Int. J. Dev. Biol. 49: 269-277 (2005)

doi: $10.1387 / \mathrm{ijdb} .041948 \mathrm{tj}$

\title{
Tracing the hemangioblast during embryogenesis: developmental relationships between endothelial and hematopoietic cells
}

\author{
THIERRY JAFFREDO*,1, KARINE BOLLEROT' ${ }^{1}$, DAISUKE SUGIYAMA ${ }^{1,3}$, RODOLPHE GAUTIER $^{1}$ \\ and CÉCILE DREVON ${ }^{1,2}$ \\ ${ }^{1}$ UMR7622, UPMC Paris VI, Paris, France, ${ }^{2}$ Université des Sciences et Technologies de Lille, Laboratoire de Biologie du Développement, \\ Villeneuve d'Ascq, France and ${ }^{3}$ The Institute of Medical Science, University of Tokyo, Tokyo, Japan
}

\begin{abstract}
We review here the development of the hematopoietic system and its relationship to the endothelium, with a special focus on the characterisation of the hemangioblast, the putative ancestor for endothelial cells and hematopoietic cells. Using the avian model, we have traced in vivo the progeny of embryonic endothelial cells and shown that aortic-born hematopoietic cells (known to generate the definitive hematopoietic lineage) derive from endothelial cells in the floor of the aorta. During this process, endothelial cells undergo a switch from endothelial cells to hematopoietic cells characterised by a downgrading of endothelial cell-specific genes and the parallel upgrading of hematopoietic cell-specific genes. Using a similar approach, we have shown that generation of hematopoietic cells from endothelial cells also takes place during mouse embryonic development. We have thoroughly characterised the dynamics of key molecules (several of which we have cloned) specifically expressed by the yolk sac or aortic hemangioblast. The yolk sac hemangioblast is characterized by the specific expression of SCL/Tal-1 and Lmo2, whereas the aortic hemangioblast expresses Runx-1 (a runt domain transcription factor). Finally, we have demonstrated the existence of a new site for hematopoiesis, namely the allantois. Using quail/chick grafts, we show that this embryonic appendage autonomously produces endothelial cells and hematopoietic cells, these latter being endowed with the attributes of the definitive hematopoietic lineage.
\end{abstract}

KEY WORDS: allantois, avian embryo, mouse embryo, endothelium, HSC, GATA, VE-Cadherin

Developmental bases of hematopoietic cell emergence and migration, an introduction

Based on observations performed quite a century ago on avian embryos, several authors attributed a central role to the Yolk Sac (YS) in the production of Hematopoietic Cells (HC). YS-produced hematopoiesis largely consists in erythroid cells that appear red due to the presence of hemoglobin. Analysing sections of embryos from various species, other authors reported at the same time the presence of cells, possibly of hematopoietic origin, associated with the ventral aspect of the aorta. Fifty years later, the role of the YS in the production of $\mathrm{HC}$ was analysed by a series of experiments conducted by Moore and Owen. The experimental scheme used observations on parabiosed embryos or chorioallantoic grafts of organ rudiments (Moore and Owen, 1965, Moore and Owen, 1967a) following exchanges of cells between male/female partners, by means of the pair of heterochromosomes, typical of the female sex in chicken, which became identifiable in cells from colchicinetreated embryos arrested in metaphase. This model yielded the founding experiments of the hematogenous theory (Moore and Owen, 1967b). These conclusions were twofold. The hematopoietic organ rudiments, i.e. the fetal liver, bone marrow and spleen, were colonized by cells coming from elsewhere. This conclusions were confirmed and extended, with the quail chick/marker leading to the fine-tuning dissection of thymic and bursa of Fabricius colonisation (Jotereau and Le Douarin, 1982, Le Douarin et al., 1984a, Le Douarin et al., 1984b). The YS produced a stock of HC part of which would differentiate in situ, while the others would colonize the hematopoietic organ rudiments (Moore and Owen, 1967b). A few years later, F. Dieterlen and co-workers initiated a series of elegant experiments that were going to modify in depth our vision of $\mathrm{HC}$ production. These experiments relied on the use of the quail/chick system combined with the accessibility and topology of the avian embryo, which lies flat upon the spherical YS. This consisted in grafting a quail embryo onto a chick YS, an

Abbreviations used in this paper: $\mathrm{AO}$, area opaca; $\mathrm{BI}$, blood islands; EC, endothelial cell; HC, hematopoietic cell; YS, yolk sac.

\footnotetext{
*Address correspondence to: Dr. Thierry Jafredo. UMR7622, UPMC Paris VI, 9 Quai St Bernard, 75005 Paris, France. Fax: +33-1-4427-3497. e-mail: jaffredo@ccr.jussieu.fr
} 
experimental schedule designated as YS chimeras, and analysing the constitution of the hematopoietic organs for the presence of quail or chick cells (Dieterlen-Lievre, 1975). Hematopoietic organs within the embryo were seeded exclusively by quail cells. Contrary to the centripetal flow of $\mathrm{HC}$ postulated by Moore and Owen, cells from the embryo even seeded the YS by embryonic day (E) 5 (Beaupain et al., 1979a, Martin et al., 1978). At E13, 70\% of circulating blood cells were of quail origin (Beaupain et al., 1979a). Similar experiments were repeated with congenic strains of chicks differing either by their sex chromosomes (Lassila et al., 1978a, Lassila et al., 1978b), their immunoglobulin haplotypes (Lassila etal., 1979, Martin etal., 1979) or major histocompatibility antigens (Lassila et al., 1982). Similar conclusions were drawn. YS cells decreased rapidly from E5 and disappeared completely at hatching. Thus, yolk sac $\mathrm{HC}$ did not display long-term renewal whereas the source of "definitive" Hematopoietic Stem Cells (HSC) colonising the hematopoietic organs originated from the embryo proper. These experiments also revealed that the YS contributes to two distinct generations of red cells: a primitive one that derives entirely from the $\mathrm{YS}$ and a second one (fetal) that derives both from the embryo and the yolk sac, the latter being able to generate erythrocytes with a fetal globin make up (Beaupain et al., 1979a, Beaupain et al., 1979b). The time- and typerestricted differentiation capacities of YS HC probably reflect the influence of the YS microenvironment that may trigger $\mathrm{HC}$ differentiation into erythroid and myeloid lineages. In this regard, when the extraembryonic area of an E2 quail embryo was associated in vitro with an E6 chick thymus, quail $\mathrm{HC}$ colonized the thymus rudiment and underwent lymphoid differentiation (Jotereau and Houssaint, 1977) meaning that the potential is present but masked by the microenvironment. Finally, using reversed YS chimeras (chick embryo grafted onto a quail YS), combined with the use of cell type- and species-specific monoclonal antibodies QH1/MB1 (Pardanaud etal., 1987, Peault et al., 1983), which recognise quail hematopoietic and Endothelial Cells (EC), it was shown that YS produced an early and small population of $\mathrm{HC}$ with characteristics of primitive macrophages, found in zones of apoptosis, which may represent the first microglial cells (Cuadros etal., 1992, Cuadros and Navascues, 2001). Such a YS contribution was also recently demonstrated for the mouse embryo (Alliot et al., 1999).

Aiming at identifying the intraembryonic site(s) where HSC were produced, Dieterlen-Lièvre and co-workers focused their attention on the aortic-associated clusters of cells which protrude into the aortic lumen. These cells were reported to display several attributes of $\mathrm{HC}$ among which a high nuclear to cytoplasm ratio and an affinity for basophilic stains ((Dieterlen-Lievre and Martin, 1981). Later on, their hematopoietic nature was confirmed with $\mathrm{MB1} / \mathrm{QH} 1$. The aortic region, (eventually designated in the mouse embryo as the PSp/AGM region for 'Para-aortic Splanchnopleura/ Aorta-Gonad-Mesonephros', i.e., the aorta and surrounding tissues at two different stages of development), was shown to harbour transplantable HSC in the avian embryo (Lassila et al., 1980). In birds, the aorta exhibits two different aspects of hematopoiesis; the first occurs during the third day of incubation (E3=Hamburger and Hamilton $-\mathrm{HH}$ - stages 17 to 21) (Hamburger and Hamilton, 1951) and is designated as the intra-aortic clusters (see above) (Fig. 1). The second aspect, designated as para-aortic foci, developing between E6.5 and E9 outside the aorta proper (Fig. 1) consists in large groups of cells distributed in the loose mesenchyme ventral to the vessel (Dieterlen-Lievre and Martin, 1981). Whereas a few cells harbouring $\mathrm{HC}$ characteristics were reported to be carried by a homologous site in the mouse embryo the existence of this second aspect has not been confirmed in mammals (see Godin and Péault, 2005 in this issue). When dissociated into individual cells and seeded into a semi-solid medium supplemented with appropriate cytokines, chick aortic cells displayed erythroid, monocytic, myeloid or multipotential colonies (Cormier, 1993, Cormier et al., 1986, Cormier and Dieterlen-Lievre, 1988).

\section{Hemangioblast and the hemogenic endothelium}

When YS BI form, $\mathrm{HC}$ and $\mathrm{EC}$ emerge at the same time in close association. In the aorta, $\mathrm{HC}$ and EC are intimately associated. The association between these two cells types has prompted earlier embryologists to assume the existence of a putative ancestral progenitor between $\mathrm{EC}$ and $\mathrm{HC}$ called the hemangioblast (Murray, 1932). This hypothesis has been reinforced by the fact that $1^{\circ}$ both cell types shared several markers and $2^{\circ}$ several gene mutations and deletions in zebrafish and mouse embryos affects both cell types. Whereas the existence of this elusive cell type has been demonstrated in ES cell cultures, such a common ancestor has not been evidenced yet in vivo. Some avian data even argue against this hypothesis; the avian homologue of VEGF-R2, Quek1 , was shown to be expressed by the mesodermal progenitors giving rise to YS mesoderm. Aiming to identify the hemangioblast, Eichmannn and co-workers (Eichmann et al., 1997) sorted out Quek-1+ cells from the very early blastodisc and submitted the positive cell population to a clonal differentiation assay. In the absence of growth factors, VEGF-R2+ cells gave rise to hematopoietic colonies. When VEGF was added, endothelial colonies differentiated while the number of hematopoietic colonies decreased substantially. Mixed colonies, which would be the proof for the hemangioblast, never developed precluding the demonstration of a common precursor. Recent mapping experiments in the mouse embryo even suggest that $\mathrm{HC}$ and $\mathrm{EC}$ are segregated separately during the gastrulation process; $\mathrm{HC}$ passing through the PS first followed by the EC population (Kinder et al., 1999).

While filiation between $\mathrm{HC}$ and $\mathrm{EC}$ is not solved yet for the $\mathrm{YS}$ $\mathrm{BI}$, generation of $\mathrm{HC}$ in the aortic region has been analysed in much more detail and is the subject of active research. In the E3 avian aorta ( $\mathrm{HH} 17$ to 21$), \mathrm{HC}$ appear to delaminate from the aortic floor endothelium. Similar groups of cells have been described in the same location in all vertebrate species investigated so far. Under the impulse of F. Dieterlen, we decided to thoroughly analyse the origin of avian intraaortic clusters and paraaortic foci with a particular focus on their putative relationship with the endothelium. We first analysed the characteristics of the cells lining the aortic lumen at the time of hematopoietic emergence (Jaffredo et al., 1998), using double staining with antibodies directed against CD45, the pan leukocyte antigen and VEGF-R2 specific for EC at that time. Before cluster emergence, aortic EC were entirely positive for VEGF-R2. When clusters differentiate, VEGF-R2 was downgraded and CD45 upgraded in EC of the ventral endothelium (Fig. 1E). As hematopoietic cells bulge in the aortic lumen, all rounded cells became CD45+ (Jaffredo et al., 1998). 

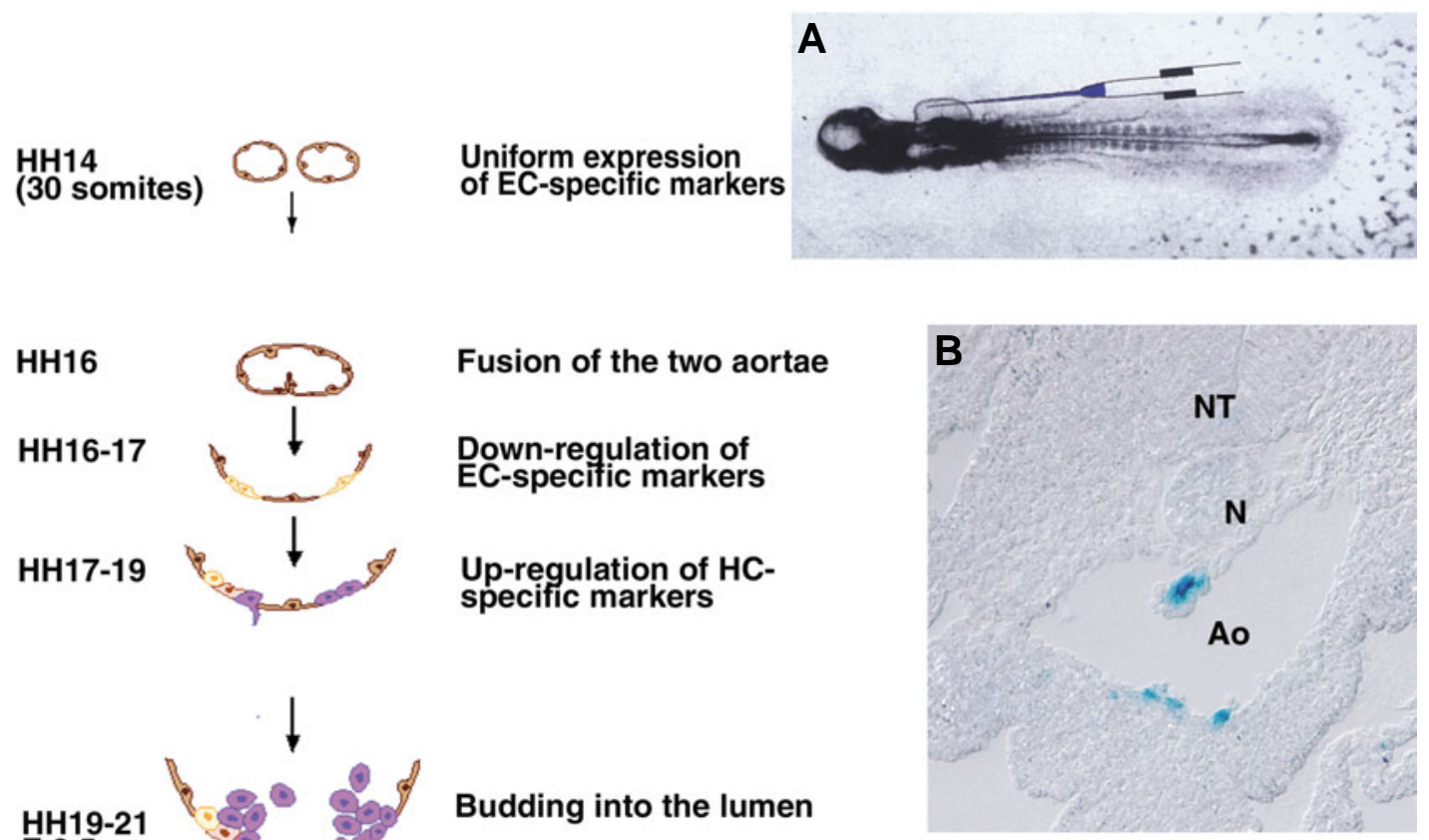

Up-regulation of HC-
specific markers

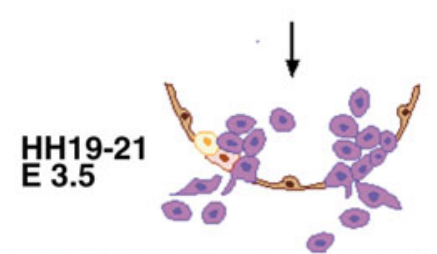

Budding into the lumen

Ingression into the mesentery
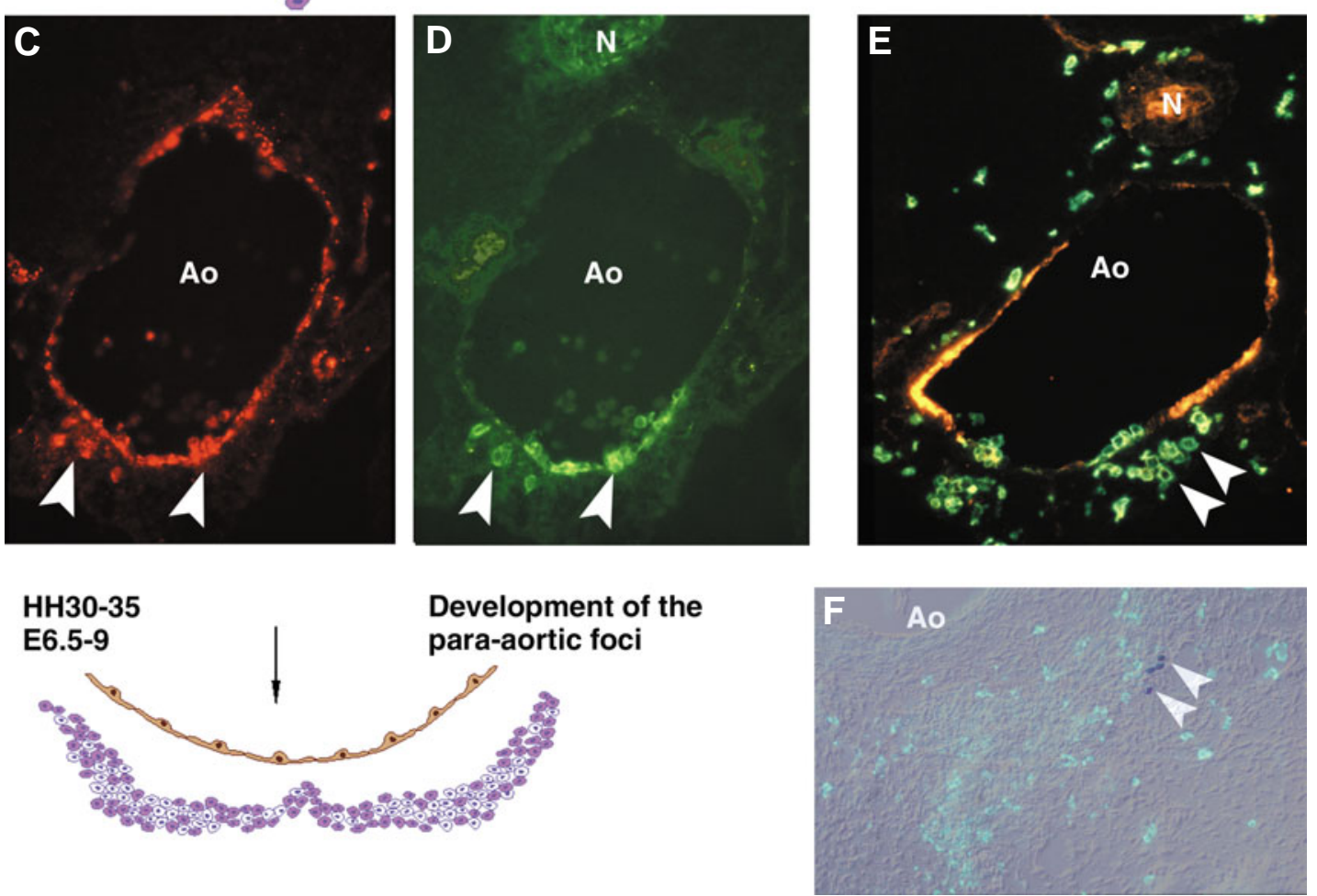

Fig. 1. Emergence of hematopoietic clusters in the aortic endothelium. The drawings represent the different steps from the paired aortae to the paraaortic foci. (A) Route of inoculation. AcLDL-Dil and non-replicative retroviral vectors are delivered into the heart with a micropipette so that the probes are in direct contact with endothelial cells lining the vessels. (B) Cross section at the mid-trunk level. lacZ staining following non-replicative retroviral vectors. 12 hours after inoculation, a few hours after fusion of the paired aortae. Both endothelial cells in the ventral and dorsal sides of the vessel are labelled. (C,D) Cross section at the mid-trunk level. Double staining for AcLDL-Dil and CD45. 24 hours after AcLDL-Dil delivery. C: endothelial cells of the aorta are strongly positive for AcLDL-Dil. Note the presence of AcLDL-Dil + budding cells as well as cells into the mesentery (arrowheads). D: Double staining with anti-CD45 which labels the hematopoietic clusters. (E) Double staining with anti-VEGF-R2 (orange) and CD45 (green). Note the presence of green cells inserted into the endothelium and accumulating into the mesentery (arrowheads). (F) Cross section at the mid-trunk level. Six days following inoculation of non-replicative vectors carrying lacZ. Double staining for CD45 (green) and lacZ (blue). Blue cells are found inside the CD45+ population thus derive from cells stained earlier on at the level of the endothelium. Abbreviations: Ao, aorta; N, notochord; NT, neural tube. 


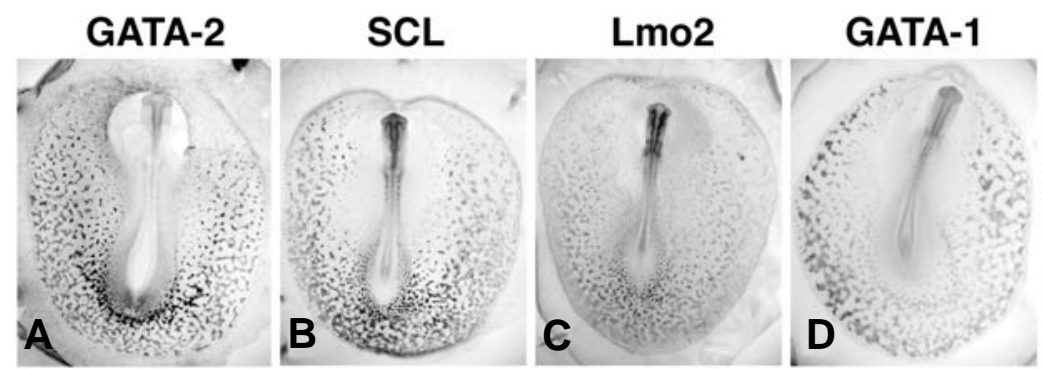

\section{9 somites}

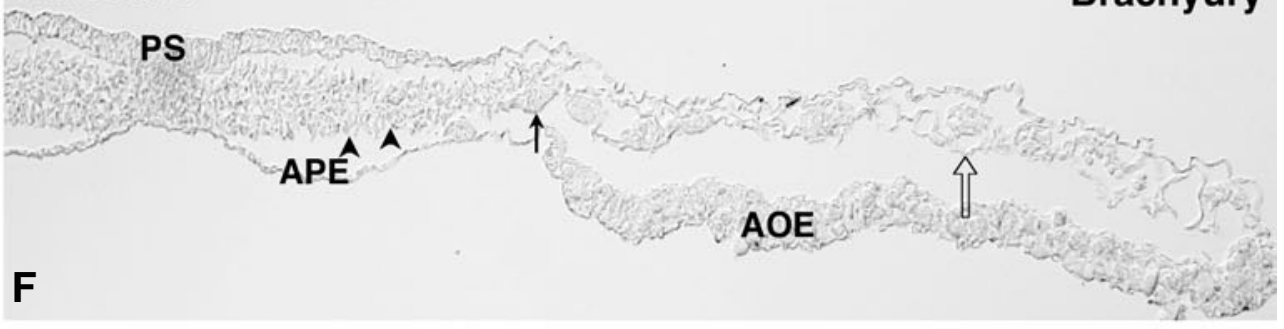

GATA-2
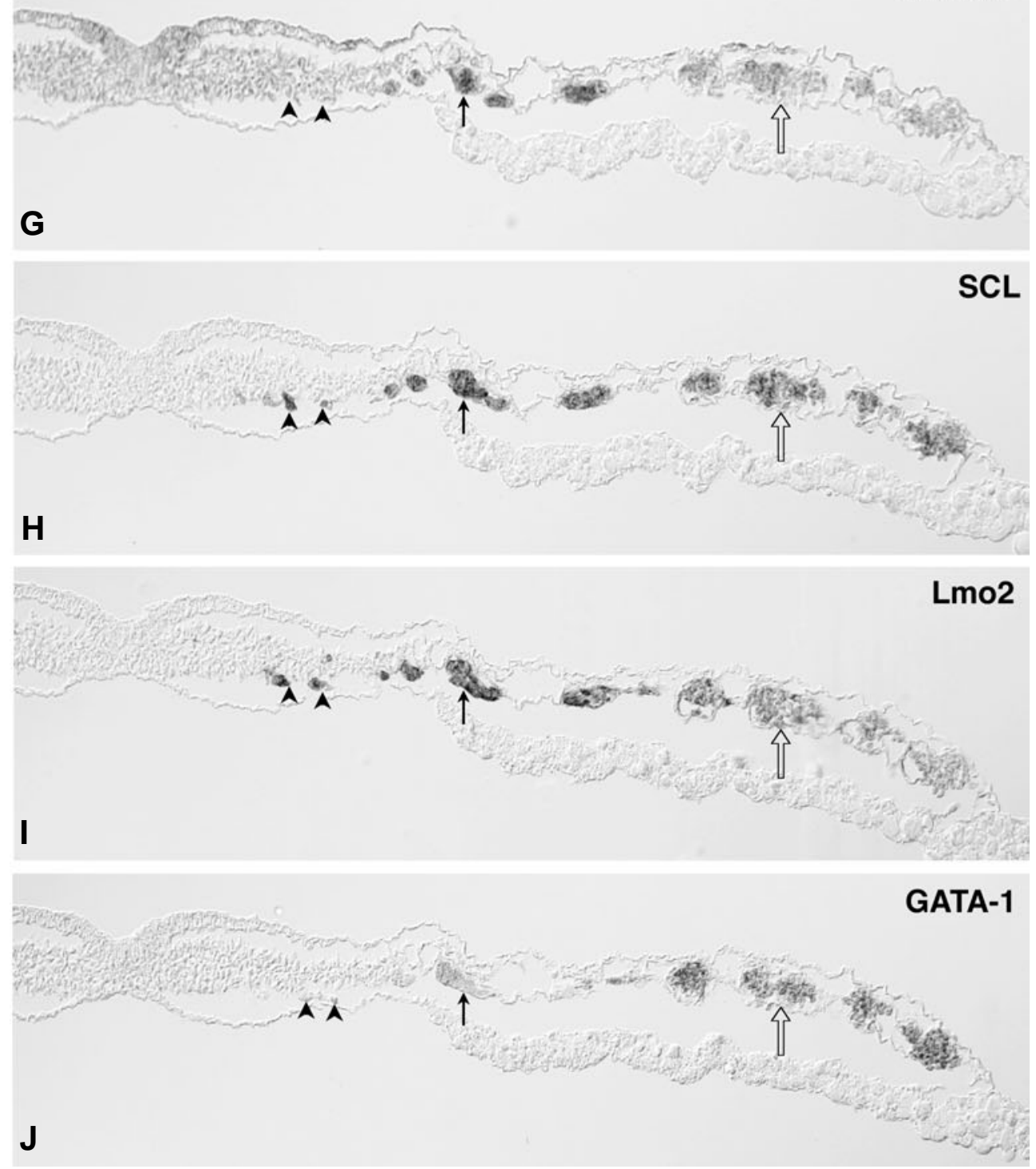

In order to obtain a dynamic approach of the developmental relationships between cluster cells and the aortic endothelium, we used two vital tracers, acetylated Low Density Lipoprotein (AcLDL) coupled to a fluorescent lipophilic marker (Dil) and non-replicative retroviral vectors carrying reporter genes. The first provides a short term tracing whereas the second allows the tagged cells to be followed in the long term. Both were introduced by intracardiac inoculation (Fig. 1A), a technique that distributes the tracers in direct contact with the endothelium that lines the vessels. Inoculations were performed in the beginning of the second day of incubation, i.e., one day before the

Fig. 2. 9-somite stage: from mesoderm to mature blood cell groups. (A-E) 9somite stage, whole mounts. (F-J) Adjacent sections, in situ hybridization. (A) GATA-2 expression has progressed throughout the AO except for its anterior-most aspect. (B) $\mathrm{SCL}$ is strongly expressed by $\mathrm{Bl}$ as well as around the posterior part of the embryo. (C) Lmo2 displays a similar pattern. (D) GATA1 , present at the level of the islands, is enhanced in lateral BI. (E) hemoglobin is now conspicuous in lateral Bl. Bar, $2 \mathrm{~mm}$. (F) Brachyury is restricted to mesoderm at the level of the streak proper. (G) GATA-2 is expressed by the mesoderm starting from its ingression through the streak. The signal is enhanced in nascent Bl at the AP/ $A O$ frontier but decreased in lateral, mature BI. (H) SCL is expressed by single cells or groups of cells, close to the endoderm adjacent from the streak (arrowheads). Expression persists in nascent BI (solid arrow) and in mature BI (empty arrow) although expression levels vary from cell to cell. (I) Lmo2 expression resembles that of $\mathrm{SCL}$. Note the enhanced expression in the external cells of the maturing $B /$ pointed out by the solid arrow. (J) GATA-1 is found in nascent B/ where GATA-2 is upregulated (compare $G$ to J). The GATA-1 signal increases as Bl mature, the highest expression level being found in the most lateral BI. Arrowheads, solid arrows and emptyarrows pointout the same structures on the different sections. Bar, $100 \mu \mathrm{m}$. Reprinted with permission from Gene Expresssion Patterns, (2003) Minko, K., Bollerot, K., Drevon, C., Hallais, M.F. and Jaffredo, T. "From mesoderm to blood islands: patterns of key molecules during yolk sac erythropoiesis." pp. 261-272. 
emergence of the first aorta-associated HC. Identification of the cells was achieved using the anti-VEGF-R2 (Eichmann et al., 1997) and the anti-CD45 antibodies (Jeurissen and Janse, 1998) as, respectively a EC- and HC-specific probe. AcLDL-Dil has two major advantages: $1^{\circ}$ it is endocytosed by EC and macrophages (which are not present in the embryo at that stage) via a specific receptor. $2^{\circ}$ It displays a short half life and rapidly disappears from the circulation even if endocytosis does not occur. As soon as two hours after inoculation, the whole vascular tree was labelled. The aorta, still paired at that time, was entirely lined by LDL+ EC while no CD45+ cell was present neither at the level of the aorta nor in the circulation. Twenty four hours after inoculation, the paired aortae have fused. LDL stain was found in EC but also in aortic clusters which displayed a strong LDL signal (Fig. 1C). Moreover LDL+/CD45+ cells were found in the mesenchyme ventral to the aorta (Fig. $1 \mathrm{C}, \mathrm{D}$ ) suggesting that a subset of $\mathrm{HC}$ ingress into this region (Jaffredo et al., 1998). Thus, intra-aortic clusters derived from cells located in the aortic floor and endowed with a typical endothelial phenotype at the time of inoculation, underwent 'transdifferentiation'. Importantly it was recently demonstrated in mouse and humans embryos that aortic $\mathrm{EC}$ can also give rise to HC (de Bruijn et al., 2002, Oberlin et al., 2002).

Contrary to intra-aortic clusters, para-aortic foci develop in mesenchyme ventral to the aorta and bear no conspicuous relationship with the aortic endothelium. Experimental analysis has shown that, among these cells, HSC are present. Is there a developmental relationship between the two aspects of aortic hemopoiesis or did they belong to two independent generations of HSC? This question is important since adults seem no longer capable of producing HSC from EC. In other terms are HSC obviously generated through an endothelium intermediate during early development or could they be generated de novo from a mesenchyme? To approach this question we have used nonreplicative lacZ-bearing retroviral vectors introduced into the circulation. The advantages of this system are twofold: $1^{\circ}$ clones can be identified because integration occurs only in a few EC. $2^{\circ}$ the reporter gene is stably expressed in the progeny of infected cells and tagged cells can be traced for long periods of time. The conclusions were as follows: Similarly to AcLDL-Dil, retroviral vectors tagged exclusively EC (Fig. 1B). At E3 the labelled clones comprised either EC and $\mathrm{HC}$ but never both. Labelled cells were observed to ingress into the mesentery ventral to the aortic floor. At E7, the para-aortic foci contained numerous lacZ+ cells (Fig. 1F) (Jaffredo et al., 2000). Thus paraortic foci are seeded by progenitors derived from the E3 aortic floor through a unique event of HSC emergence from the aortic endothelium.

\section{Hemogenic endothelium in the mouse embryo}

In the mouse embryo the AGM region is also a site where definitive hematopoiesis takes place (Godin and Cumano, 2002, Ling and Dzierzak, 2002). At the same time we demonstrated the endothelial origin of the intraaortic clusters in the avian embryo, Nishikawa and colleagues (1998) showed that EC, isolated from the E9.5 mouse embryo on the basis of VE-cadherin expression, were able to give rise to blood cells in vitro. Using a fine tuning dissection approach of the AGM, it was shown that HSC originate from the dorsal aorta and the surrounding tissues (de Bruijn et al., 2000). Finally using transgenic mice carrying the GFP gene under the control of the Ly6-A promoter, the first definitive HSC were shown to appear in the endothelial layer (de Bruijn et al., 2002). The results collected from the avian embryo prompted us to apply a similar tracing approach to the mouse embryo in order to look for an endothelial origin of HSC. In collaboration with Daisuke Sugiyama, we have analysed the production of $\mathrm{HC}$ by the vesselassociated endothelium during embryonic development of the mouse. Like in the avian embryo, AcLDL-Dil was injected intracardially in cultured embryos. One hour after inoculation, Dil staining was found along the entire endothelial tree. Following dissociation, FACS analysis revealed that Dil+ cells were CD31+, CD34+ but not CD45, the antigen make-up characteristic for the endothelial lineage. Upon clonal culture, these cells gave rise to adult type, erythroid colonies that displayed an adult globin chain arrangement. Some embryos were allowed to develop for an additional twelve or twenty four hours. Twelve hours after inoculation, $43 \%$ of Dil+ circulating cells belonged to the definitive erythroid lineage. Beyond the 29-somite stage, the proportion of the Dil+ erythroid cells gradually decreased. These results demonstrate, for the first time in the mouse embryo, the generation of hematopoietic cells from an endothelial intermediate, using an in vivo tracing. We thus provide evidence for the release of these cells into the circulation and put forward the hypothesis that they are able to colonize the fetal liver and generate definitive erythrocytes in vivo (Sugiyama et al., 2003).

\section{Molecular characterisation of the hemangioblast and the hemogenic endothelium}

The experimental demonstration of hemogenesis by the aortic endothelium has prompted us to seek for molecules specifically expressed by the hemangioblast or the hemogenic endothelium. Using molecular markers, several of which we have cloned, we have been able to identify steps from the deposition of the mesoderm to $\mathrm{EC}$ and $\mathrm{HC}$ in the $\mathrm{YS}$ and at the level of the aorta in the transition from $\mathrm{EC}$ to $\mathrm{HC}$. The blood-forming system, namely $\mathrm{EC}$ and $\mathrm{HC}$, differentiates from the mesoderm during the gastrulation process, i.e., the ingression of cells from the superficial epiblast. Extraembryonic mesoderm is deposited first and contributes to the Area Opaca $(\mathrm{AO})$, so-called because cells constituting the endodermal layer, the hypoblast, are yolky thus look opaque. Mesoderm giving rise to $\mathrm{BI}$ was shown to be deposited at early stages from cells located in the posterior part of the primitive streak (Schoenwolf et al., 1992). A mapping of the primitive streak in the mouse embryo recently yielded similar conclusions (Kinder et al., 1999). Cells first deposited undergo extensive lateralwards and cephalwards migrations and reach the edges of the blastodisc, while cells passing through the streak immediately after occupy more medial and more caudal positions. Thereafter, cells gradually fill more central and more posterior positions as the primitive streak regresses from cephalic to caudal levels. Thus, early deposited cells have a longer life history than their immediate neighbours and undergo differentiation earlier. Blood Islands, wherefrom the first $\mathrm{HC}$ are going to emerge, differentiate from the $\mathrm{AO}$ mesoderm. $\mathrm{BI}$ are typically constituted of an outer layer of EC and a core of $\mathrm{HC}$. $\mathrm{BI}$ will progressively spread onto the entire YS. Mesoderm giving rise to the embryonic area, designated as the Area Pellucida (AP), because it lies above the segmentation cavity, thus appears clear. The AP is generated by cells occupying a more anterior position 

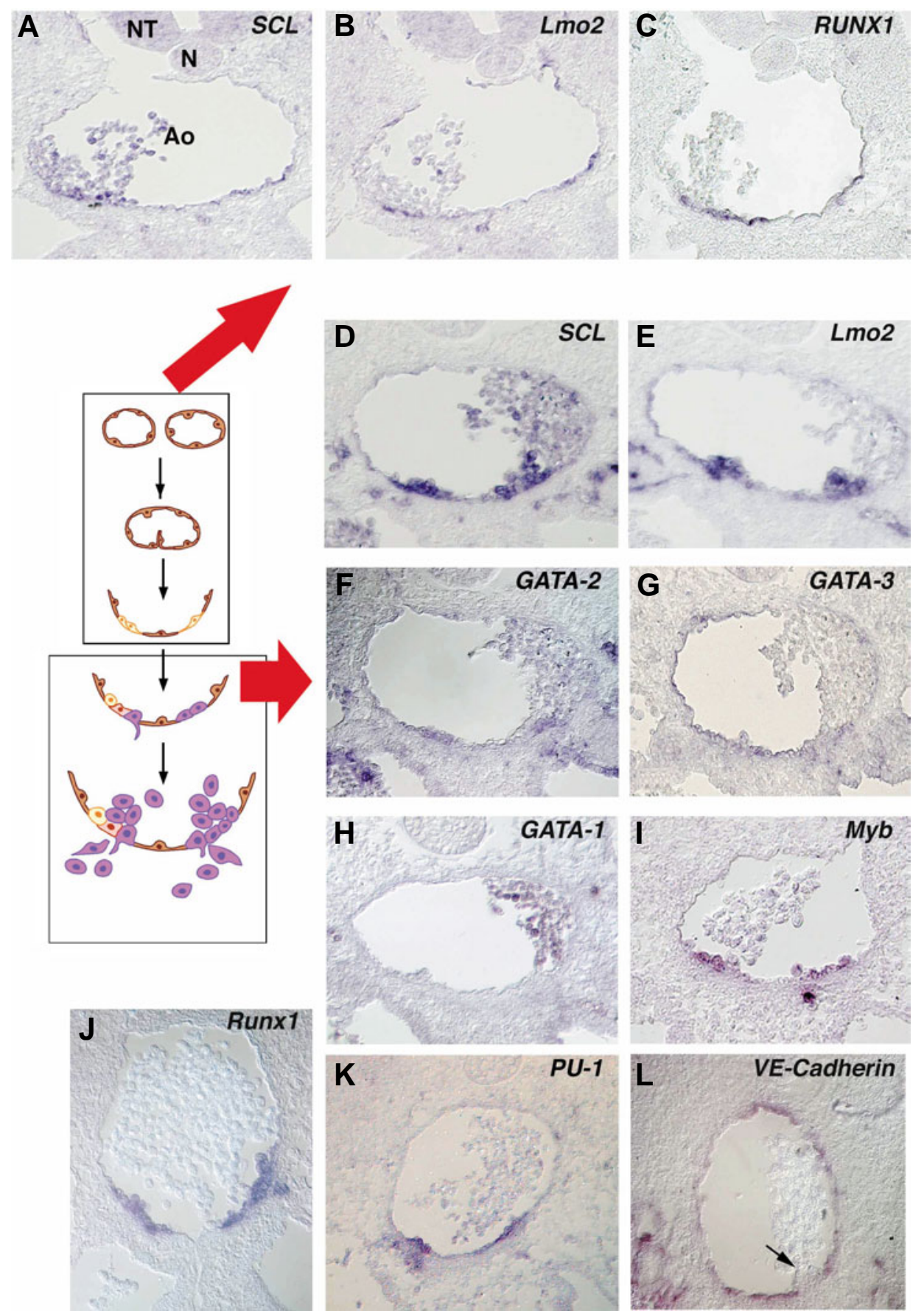

Fig. 3. Gene activity during formation of the intraaortic clusters. The drawing in the left side schematises the different steps in the formation of the clusters. The frames point out the two major aspects of cluster formation: hemogenic endothelium without any morphological sign of hematopoiesis and full cluster formation. (A-C) Beginning of the third day of development when intraaortic clusters have not appeared yet. Adjacent sections. (D-L) Middle of the third day when intraaortic clusters are fully developed. (A) SCL/Tal-1 is expressed by all aortic EC but appears strengthened in the aortic floor. Note the presence of SCL/Tal-1+ circulating cells. (B) Lmo2 stains all aortic EC. A few circulating cells are Lmo2+. (C) Runx1 expression is restricted to EC of the aortic floor. (D) SCL/Tal-1 is now vividly expressed by the clusters and expressed at low levels by aortic EC. (E) Lmo2 displays a similar signal. In addition non-aortic EC display a conspicuous Lmo2 expression. Reprinted with permission from Hematopoietic stem cells, Dr. I. Godin and A. Cumano editors; Extra and intraembryonic HSC commitment in the avian model. Jaffredo T., Bollerot K., Minko K., Gautier R., Romero S and Drevon C. Copyright 2003 with permission from Landes Biosciences.

along the primitive streak (Schoenwolf et al., 1992). At the time BI differentiate, the AP, wherefrom the embryo proper develops, do not participate to this early blood-forming phase, however it contains structures very similar to $\mathrm{YS} \mathrm{BI}$ (referred here as vascular islands) that never develop hematopoiesis. The bloodforming potential of this latter area will be revealed two days later as the aortic region will undergo hematopoiesis. We have probed these early events by examining the patterns of several molecules likely to be involved in $\mathrm{HC}$ and EC emergence. The molecules analyzed are genes involved in cell fates choices i.e., Notch and its ligands and transcription factors which participate in multimeric complexes: GATA-1, -2, -3, SCLI Tal-1 and $\angle m O 2$, (whose avian orthologue we have cloned) and VEGF-R2, a regulator of hematopoietic and endothelial commitment. Notch signalling is an important evolutionary conserved mechanism known to control cell fates choices through local interactions. Here, the patterns of expression of Notch-1 and -2 genes and their ligands, Delta-1, Serrate-1 and -2 , were established in the early blastodisc of the chicken embryo from the pre-streak to the first somite stages. Delta-1 was detected as early as the pre-streak stage in the posterior part of the embryo shortly followed in the same region by Notch-1at the initial streak stage. Thereafter both were strongly expressed in the posterior part of the primitive streak until HH4. Notch-2 was also found at the level of the streak although at low levels. Notch-1 was homogeneously expressed by the epiblast and by mesodermal cells ingressing at the level of the streak whereas Delta-1 expression formed a "salt and pepper" pattern. No Notch and Delta activity was found in the YS blood islands suggesting that lineage restriction may occur very early during development (Caprioli et al., 2002). As soon as gastrulation proceeds at $\mathrm{HH} 3$ and $\mathrm{HH} 4$ (respectively 12-13h and $18 \mathrm{~h}$ of incubation) GATA-2 and VEGF-R2 were detected the earliest in the whole ingressing mesoderm. Two distinct patterns were evident; one low, associated to the ingressing mesoderm, one high, specific, restricted to mesodermal cells in direct contact with the endoderm although no morphologic segregation was visible in the mesodermal 
compartment. We interpret this pattern as an early commitment event resulting from endoderm/mesoderm interactions. From this time SCL/Tal-1 and Lmo2 expression appeared in groups of cells or single cells likely to be hemangioblasts, which shared expression of GATA-2 and VEGF-R2 with the rest of the mesoderm. This is followed by the upregulation of GATA-2 in hemangioblastic aggregates (Fig. $2 \mathrm{~A}$ to $\mathrm{J}$ ). These gene expressions were preferentially found in cells located in the ventral aspect of the mesoderm and were accompanied by the appearance of morphologically distinct structures. At later stages, these structures became more conspicuous accompanied by the expression of SCL/Tal1, Lmo2 and the appearance of GATA-1 (Minko et al., 2003).

Gene activities have been also thoroughly characterized at the level of the aorta. When the aortae are still paired and no sign of hematopoiesis is visible, aortic EC express molecules characteristic of an endothelium (the surface molecules VEGF$\mathrm{R} 2, V E$-Cadherin and the low expression of transcription factors Lmo2, SCL/Tal-1). When the aortae were still paired or when they underwent fusion, Runx 1 and GATA-2 expression appeared whereas $S C L / T a /$-1 and $L$ mo2, became upregulated in the ventral aspect of the aorta, that at the same time retained expression of the EC-specific genes (Fig. 3A, B, C). The steps after fusion were characterized by the thickening of ventral EC accompanied by the increased expression of $\mathrm{HC}$-specific genes and the decrease of EC's (downgrading of VEGF-R2, VE-Cadherin upgrading of Runx1, SCL/Tal-1, Lmo2, GATA-2, GATA-3). From that time, hematopoietic clusters became conspicuous and expressions of $\mathrm{EC}$ and HC-specific genes were dissociated (Fig. 3D to L). Runx1, the avian orthologue of which we have cloned, is the only gene whose expression to date is known to be restricted to the ventral region of the aorta. This transcription factor contains an evolutionarily conserved DNA-binding domain termed the runt domain which is also required for heterodimerisation with a cofactor termed $C B F \beta$. Inactivation of this gene in the mouse embryo precludes the emergence of the definitive hematopoiesis (Wang etal., 1996). Using a knock in approach, Runx 1 was shown to be specifically expressed by the ventro-lateral aortic EC, by the intra-aortic clusters (North et al., 1999) and was also reported to be tightly associated to the HSC fraction (North etal., 2002). In the avian embryo Runx 1 is expressed as early as E2.5 in EC of the ventral aortic floor. Expression begins when the aortae are still paired and persisted upon fusion of the vessels. At the time of hematopoietic cluster production, Runx 1 becomes restricted to $\mathrm{HC}$ buddging into the lumen and ingressing into the mesentery. Interestingly $P U-1$, a putative target gene for Runx 1 , expressed in precursors of the myeloid and lymphoid lineages, is detected in intra-aortic clusters (Fig. 3).

\section{The allantois: another source for hemangioblasts?}

In birds, the timing of intra-aortic clusters and para-aortic foci is compatible with the seeding of the thymus and bursa of Fabricius. Cells of the para-aortic foci have been shown to have $B$ and $T$ lymphoid potentials. However, seeding of the bone marrow begins by $\mathrm{E} 10.5$ in the chick embryo and continues until birth. At this time, the activity of the para-aortic foci has ceased. The possibility that cells from the para-aortic foci remained in an undifferentiated state somewhere into the embryo appeared unlikely. We thus have looked for another progenitor-producing site and identified the allantois. This organ has the appropriate tissue make-up to produce hemopoiesis i.e., endoderm associated to mesoderm. This appendage was shown to be involved in gas exchange, excretion product accumulation, shell $\mathrm{Ca}++$ resorbtion and bone construction. By using India ink or AcLDL-Di microangiographies, we first established that the allantois became vascularized between 75-80 hours of incubation. It displayed conspicuous red cells even before this period indicating that hematopoiesis occurred independently from the rest of the embryo in this site (Caprioli et al., 1998, Caprioli et al., 2001). The allantois undergoes a program characterized by the prominent expression of several "hemangioblastic" genes in the mesoderm accompanied by other gene patterns in the associated endoderm. VEGF-R2, at least from stage $\mathrm{HH} 17$ onward, is expressed and is shortly followed by transcription factors GATA2, SCL/Tal-1and GATA-1. Blood island-like structures differentiate that contain both CD45+ cells and cells accumulating hemoglobin; these structures look exactly like blood islands in the yolk sac. This hemopoietic process takes place before the establishment of a vascular network connecting the allantois to the embryo. As far as the endoderm is concerned, GATA-3mRNA is found in the region where allantois will differentiate before the posterior instestinal portal becomes anatomically distinct. Shortly before the bud grew out, GATA-2 was expressed in the endoderm and, at the same time, the hemangioblastic program became initiated in the mesoderm. GATA-3 was detected at least until E8 and GATA-2 until E3 the latest stage examined for this factor. Using in vitro cultures, we showed that allantoic buds, dissected out before the establishment of circulation between the bud and the rest of the embryo, produced erythrocytes of the definitive lineage. Moreover, using heterospecific grafts between chick and quail embryos, we demonstrated that the allantoic vascular network develops from intrinsic progenitors (Caprioli et al., 2001). Taken together, these results extend our earlier findings about the commitment of mesoderm to the endothelial and hemopoietic lineages in the allantois. The detection of a prominent GATA-3 expression restricted to the endoderm of the preallantoic region and allantoic bud, followed by that of GATA-2, is an interesting and novel information, in the context of organ formation and endoderm specification in the emergence of hemopoietic cells.

Recent work in the mouse embryo indicates that the fetal part of the placenta that derived from the allantois is a source of hematopoietic progenitors (see Dieterlen-Lièvre et al., 2005 in this issue).

\section{Acknowledgements}

We thank DrF. Dieterlen for constant support throughout this work. We also wish to thank A. Caprioli and K. Minko, two former members of the laboratory, for their contributions reviewed in the present article. This study was supported by the Centre Nationalpourla Recherche Scientifique, by grants from the Association pour la Recherche Contre le Cancer $N^{\circ} 5131$, from the Fondation pour la Recherche Médicale and by an MERT $A C I N^{\circ} 22-2002-296 . K B$ is recipient of a French MERT fellowship $N^{\circ}$ 00391.

\section{References}

ALLIOT, F., GODIN, I. and PESSAC, B. (1999). Microglia derive from progenitors, originating from the yolk sac and which proliferate in the brain. Brain Res Dev Brain Res 117: 145-52. 
BEAUPAIN, D., MARTIN, C. and DIETERLEN-LIEVRE, F. (1979a). Are developmental hemoglobin changes related to the origin of stem cells and site of erythropoiesis? Blood 53: 212-25.

BEAUPAIN, D., MARTIN, C. and DIETERLEN-LIEVRE, F. (1979b). Origin and evolution of hemopoietic stem cells in avian embryo. In Hemoglobins in development and differentiation, (ed. STAMMATOYANOPOULOS, G. and NIENHUIS, A.). Alan R Liss, Inc., New York, pp.161-169.

CAPRIOLI, A., GOITSUKA, R., POUGET, C., DUNON, D. and JAFFREDO, T. (2002). Expression of notch genes and their ligands during gastrulation in the chicken embryo. Mech Dev 116: 161-4.

CAPRIOLI, A., JAFFREDO, T., GAUTIER, R., DUBOURG, C. and DIETERLENLIEVRE, F. (1998). Blood-borne seeding by hematopoietic and endothelial precursors from the allantois. Proc Nat/ Acad Sci Y $\Sigma A$ 95: 1641-6.

CAPRIOLI, A., MINKO, K., DREVON, C., EICHMANN, A., DIETERLEN-LIEVRE, F. and JAFFREDO, T. (2001). Hemangioblast commitment in the avian allantois: Cellular and molecular aspects. Dev Biol 238: 64-78.

CORMIER, F. (1993). Avian pluripotent haemopoietic progenitor cells: Detection and enrichment from the para-aortic region of the early embryo. J Cell Sci 105 : 661-6.

CORMIER, F., DE PAZ, P. and DIETERLEN-LIEVRE, F. (1986). In vitro detection of cells with monocytic potentiality in the wall of the chick embryo aorta. Dev Biol 118: 167-75.

CORMIER, F. and DIETERLEN-LIEVRE, F. (1988). The wall of the chick embryo aorta harbours m-cfc, g-cfc, gm-cfc and bfu-e. Development 102: 279-85.

CUAdRos, M.A., COLTEY, P., CARMEN NIETO, M. and MARTIN, C. (1992). Demonstration of a phagocytic cell system belonging to the hemopoietic lineage and originating from the yolk sac in the early avian embryo. Development 115 : 157-68.

CUADROS, M.A. and NAVASCUES, J. (2001). Early origin and colonization of the developing central nervous system by microglial precursors. Prog Brain Res 132: 51-9.

DE BRUIJN, M.F., MA, X., ROBIN, C., OTTERSBACH, K., SANCHEZ, M.J. and DZIERZAK, E. (2002). Hematopoietic stem cells localize to the endothelial cell layer in the midgestation mouse aorta. Immunity 16: 673-83.

DE BRUIJN, M.F., SPECK, N.A., PEETERS, M.C. and DZIERZAK, E. (2000). Definitive hematopoietic stem cells first develop within the major arterial regions of the mouse embryo. EMBO $J$ 19: 2465-74.

DIETERLEN-LIEVRE, F. (1975). On the origin of haemopoietic stem cells in the avian embryo: An experimental approach. J Embryol Exp Morpho/ 33: 607-19.

DIETERLEN-LIEVRE, F. and MARTIN, C. (1981). Diffuse intraembryonic hemopoiesis in normal and chimeric avian development. Dev Biol 88: 180-91.

DIETERLEN-LIEVRE, F. (2005). Commitment of hematopoietic stem cells in avian and mammalian embryos: an ongoing story. Int. J. Dev Biol 49: 125-130. doi: 10.1387/ijdb.041938fd

EICHMANN, A., CORBEL, C., NATAF, V., VAIGOT, P., BREANT, C. and LE DOUARIN, N.M. (1997). Ligand-dependent development of the endothelial and hemopoietic lineages from embryonic mesodermal cells expressing vascular endothelial growth factor receptor 2. Proc Nat/ Acad Sci Y $\Sigma A$ 94: 5141-6.

GODIN, I. and CUMANO, A. (2002). The hare and the tortoise: An embryonic haematopoietic race. Nat Rev Immunol 2: 593-604.

GODIN, I. and CUMANO, A. (2005). Of birds and mice: hematopoietic stem cell development. Int. J. Dev. Biol. 49: 251-257. doi: 10.1387/ijdb.041945ig

HAMBURGER, V. and HAMILTON, H.L. (1951). A series of normal stages in the development of the chick embryo. J. Morphol. 88: 49-92.

JAFFREDO, T., GAUTIER, R., BRAJEUL, V. and DIETERLEN-LIEVRE, F. (2000). Tracing the progeny of the aortic hemangioblast in the avian embryo. Dev Biol 224: 204-14.

JAFFREDO, T., GAUTIER, R., EICHMANN, A. and DIETERLEN-LIEVRE, F. (1998). Intraaortic hemopoietic cells are derived from endothelial cells during ontogeny. Development 125: 4575-83.

JEURISSEN, S.H. and JANSE, E.M. (1998). The use of chicken-specific antibodies in veterinary research involving three other avian species. Vet $Q$ 20: 140-3.

JOTEREAU, F. and HOUSSAINT, E. (1977). Experimental studies on the migration and differentiation of primary lymphoid stem cells in the avian embryo. In Developmental immunobiology, (ed. SOLOMON, J. B. and HORTON, J. D.). Elsevier North Holland Biomedical Press., Amsterdam.

JOTEREAU, F.V. and LE DOUARIN, N.M. (1982). Demonstration of a cyclic renewal of the lymphocyte precursor cells in the quail thymus during embryonic and perinatal life. J Immunol 129: 1869-77.

KINDER, S.J., TSANG, T.E., QUINLAN, G.A., HADJANTONAKIS, A.K., NAGY, A. and TAM, P.P. (1999). The orderly allocation of mesodermal cells to the extraembryonic structures and the anteroposterior axis during gastrulation of the mouse embryo. Development 126: 4691-701.

LASSILA, O., ESKOLA, J., TOIVANEN, P. and DIETERLEN-LIEVRE, F. (1980). Lymphoid stem cells in the intraembryonic mesenchyme of the chicken. Scand J Immunol 11: 445-8.

LASSILA, O., ESKOLA, J., TOIVANEN, P., MARTIN, C. and DIETERLENLIEVRE, F. (1978a). The origin of lymphoid stem cells studied in chick yold sac-embryo chimaeras. Nature 272: 353-4.

LASSILA, O., ESKOLA, J., TOIVANEN, P., MARTIN, C. and DIETERLENLIEVRE, F. (1978b). The origin of lymphoid stem cells studied in chick yolk sac-embryo chimaeras. Nature 272: 353-4.

LASSILA, O., MARTIN, C., DIETERLEN-LIEVRE, F., NURMI, T.E., ESKOLA, J. and TOIVANEN, P. (1979). Is the yolk sac the primary origin of lymphoid stem cells? Transplant Proc 11: 1085-8.

LASSILA, O., MARTIN, C., TOIVANEN, P. and DIETERLEN-LIEVRE, F. (1982). Erythropoiesis and lymphopoiesis in the chick yolk-sac-embryo chimeras: Contribution of yolk sac and intraembryonic stem cells. Blood 59: 377-81.

LE DOUARIN, N., JOTEREAU, F., HOUSSAINT, E. and THIERY, J.-P. (1984a). Primary lymphoid organ ontogeny in birds. In Chimeras in developmental biology, (ed. LE DOUARIN, N. L. M. and MCLAREN, A.). Academic Prss, London, pp.179.

LE DOUARIN, N.M., DIETERLEN-LIEVRE, F. and OliVER, P.D. (1984b). Ontogeny of primary lymphoid organs and lymphoid stem cells. Am J Anat 170: 261-99.

LING, K.W. and DZIERZAK, E. (2002). Ontogeny and genetics of the hemato/ lymphopoietic system. Curr Opin Immunol 14: 186-91.

MARTIN, C., BEAUPAIN, D. and DIETERLEN-LIEVRE, F. (1978). Developmental relationships between vitelline and intra-embryonic haemopoiesis studied in avian 'yolk sac chimaeras'. Cell Differ 7: 115-30.

MARTIN, C., LASSILA, O., NURMI, T., ESKOLA, J., DIETERLEN-LIEVRE, F. and TOIVANEN, P. (1979). Intraembryonic origin of lymphoid stem cells in the chicken: Studies with sex chromosome and IgG allotype markers in histocompatible yolk sac-embryo chimaeras. Scand J Immuno/ 10: 333-8.

MINKO, K., BOLLEROT, K., DREVON, C., HALLAIS, M.F. and JAFFREDO, T. (2003). From mesoderm to blood islands: Patterns of key molecules during yolk sac erythropoiesis. Gene Expr Patterns 3: 261-72.

MOORE, M.A.T. and OWEN, J.J.T. (1965). Chromosome marker studies in the development of the hematopoietic system in the chick embryo. Nature 208: 956.

MOORE, M.A.T. and OWEN, J.J.T. (1967a). Experimental studies on the development of the thymus. J. Exp. Med 126: 715.

MOORE, M.A.T. and OWEN, J.J.T. (1967b). Stem cell migration in developing myeloid and lyphoid systems. Lancet 2: 658.

MURRAY, P.D.F. (1932). The development «in vitro» of blood of the early chick embryo. Strangeways Res. Lab. Cambridge. pp. 497-521.

NORTH, T.E., DE BRUIJN, M.F., STACY, T., TALEBIAN, L., LIND, E., ROBIN, C., BINDER, M., DZIERZAK, E. and SPECK, N.A. (2002). Runx1 expression marks long-term repopulating hematopoietic stem cells in the midgestation mouse embryo. Immunity 16: 661-72.

OBERLIN, E., TAVIAN, M., BLAZSEK, I. and PEAULT, B. (2002). Blood-forming potential of vascular endothelium in the human embryo. Development 129: 4147-57.

PARDANAUD, L., ALTMANN, C., KITOS, P., DIETERLEN-LIEVRE, F. and BUCK, C.A. (1987). Vasculogenesis in the early quail blastodisc as studied with a monoclonal antibody recognizing endothelial cells. Development 100: 339-49.

PEAULT, B.M., THIERY, J.P. and LE DOUARIN, N.M. (1983). Surface marker for hemopoietic and endothelial cell lineages in quail that is defined by a monoclonal antibody. Proc Nat/ Acad Sci Y 2 A 80: 2976-80. 
SCHOENWOLF, G.C., GARCIA-MARTINEZ, V. and DIAS, M.S. (1992). Mesoderm movement and fate during avian gastrulation and neurulation. Dev Dyn 193: 235-48.

SUGIYAMA, D., OGAWA, M., HIROSE, I., JAFFREDO, T., ARAI, K. and TSUJI, K. (2003). Erythropoiesis from acetyl Idl incorporating endothelial cells at the preliver stage. Blood 101: 4733-8.
WANG, Q., STACY, T., BINDER, M., MARIN-PADILLA, M., SHARPE, A.H. and SPECK, N.A. (1996). Disruption of the cbfa2 gene causes necrosis and hemorrhaging in the central nervous system and blocks definitive hematopoiesis.

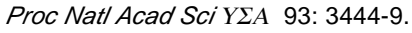

